\title{
Research Progress of Nursing Care for Elderly Patients With Complicating Postoperative Hip Fracture Deep Vein Thrombosis in China
}

\author{
Dandan Zheng ${ }^{1}$, Wentao Cheng ${ }^{2}$, Heyu $\mathrm{Wu}^{3}$, Haichao $\mathrm{Wu}^{4} \&$ Yuqi $\mathrm{Cao}^{5}$ \\ ${ }^{1}$ Postgraduate Department of Medicine, Yangtze University, Jingzhou, China \\ ${ }^{2}$ Department of Psychiatry, The Second Affiliated Hospital of Hubei University of Science and Technology, \\ Xianning, China \\ ${ }^{3}$ Operating Room, Union Hospital, Tongji Medical College, Huazhong University of Science and Technology, \\ Wuhan, China \\ ${ }^{4}$ Urology, Kunming Medical University, Kunming, China \\ ${ }^{5}$ Department of Medicine, Hubei University of Science and Technology, Xianning, China \\ Correspondence: Wentao Cheng, Department of Psychiatry, The Second Affiliated Hospital of Hubei University \\ of Science and Technology, Xianning, China. Tel: 86-153-7717-0870. E-mail: xiaoxuzong616@163.com
}

Received: November 16, 2021

Accepted: December 13, $2021 \quad$ Online Published: December 30, 2021

doi:10.20849/ijsn.v6i4.948

URL: https://doi.org/10.20849/ijsn.v6i4.948

Fund Support: [WJ2021M229 Construction and application study of a risk assessment system for deep vein thrombosis in gynecological surgery patients]

\begin{abstract}
Introduction: The aging of China's population has further deepened, and according to the results of the seventh national census, the population aged 60 years and above in China was 264.02 million, accounting for $18.70 \%$ (China.2021).The rapid growth of the elderly population has led to an increase a geriatric orthopedic morbidity. The hip fracture is one of the common orthopedic diseases, which has posed a severe threat to the elderly's life and health (Yu, 2019).

Purpose: In this study, we investigated the domestic and international literature, focusing on the analysis of DVT nursing prevention programs for elderly patients after hip arthroplasty, and proposed the current nursing research progress in preventing DVT to help elderly patients actively cope with DVT, improve the quality of life in later life, and make DVT a truly preventable disease.

Method: Electronic searches using scholarly databases were employed and only significant articles that met the review objective were utilized.

Findings: Currently, the main treatment option for hip fracture patients in clinical practice is surgery, and how to prevent Deep Vein Thrombosis (DVT) is an important task in the postoperative care of elderly orthopedic patients $(\mathrm{Li}, 2016)$. Research studies have shown that all elderly patients are at a very high risk of DVT during hospitalization, but the current trend is that $50 \%$ of patients at high risk of thrombosis are still not actively and effectively prevented from developing thrombosis for various reasons.

Conclusion: This disease, which has a high incidence, a high mortality rate, and is difficult to prevent, requires increased attention and necessitates exploration to derive effective nursing intervention programs and related nursing measures to prevent reduce the formation of DVT and alleviate suffering for patient (Xu, 2016).

In this review, we summarized the main nursing methods for preventing deep vein thrombosis of the lower extremities after hip fracture surgery in the elderly. Elderly patients are a special group of people, both physically and psychologically in a relatively fragile state, especially after the operation, so they need to be more careful in nursing work. In addition to general physical care, the care of their psychological state is also extremely important. This article describes in detail the physiological and psychological nursing interventions for patients, which can effectively guide and help medical staff to cope with the clinical care of such patients, and enable patients to better recover from surgery and trauma, and obtain a good prognosis.
\end{abstract}

Keywords: deep vein thrombosis, hip arthroplasty, orthopedic surgery, elder patients 


\section{Introduction}

\subsection{Background of the Study-Concept of DVT and Factors of Occurrence}

Deep vein thrombosis is an abnormal clotting of blood components in deep vein vessels due to various reasons. When the blood components clot, they form a small embolus that can travel the whole body with the blood flow and blocking important vascular channels. Patients then experience high fever, swelling and pain in the limbs, and numbness at the ends of the limbs.

Without timely preventive measures, as the thrombus continues to coagulate and the embolus becomes larger, the higher the risk of the patient suffering from pulmonary embolism, which can eventually lead to death (Liu, 2020; Xu, 2017). In the classification of thrombus according to the site of onset, it can be simply divided into three types: peripheral, central, and mixed (Wang, 2016). The most recognized etiology of DVT is the Virchow's triad proposed by Virchow in 1865. Virchow's triad is a series of 3 changes (Blood flow abnormality, Endothelial cell dysfunction/injury, Hypercoagulable state) that happen inside the veins (Zhuang, 2007). In addition, as research progresses, other related etiologies gradually enrich the theory.

In domestic and international studies, high age and poor lifestyle habits, as well as obesity, can directly influence and contribute to the formation of DVT in patients (KEENAN, 2007). Elderly patients require a longer recovery time after hip surgery due to the decline in physical function and underlying diseases as they enter old age. Most patients are bedridden for long periods of time during hospitalization, resulting in slow blood flow, and the endothelial damage caused by the surgery predisposes them to thrombosis. In addition, the surgical approach, site, medications, and other systemic diseases can contribute to thrombosis (GOLOMB, 2014). In elderly people who develop DVT of the lower extremities, they experience limb distension and pain as well as general discomfort, and in severe cases, the thrombus continuously coagulates and drifts in the blood vessels, leading to loss of lower extremity function, and in more severe cases, the thrombus directly obstructs the pulmonary artery leading to pulmonary is chemia and death of the patient within a short period of time (Zheng, 2017). In a study by Chen Wenyue et al. (Chen, 2020), the risk factors for DVT in Chinese patients currently undergoing artificial knee arthroplasty were systematically evaluated by Meta-analysis. In this article, a systematic search of the database for risk factors for DVT in Chinese patients undergoing hip and knee arthroplasty yielded 16 publications. The main risk factors for DVT in patients after hip and knee arthroplasty. However, there are still some limitations of the study. (Hong, 2019). hong etal used an RCT study to determine the risk factors for DVT after postoperative total joint arthroplasty (TJA). A total of 15326 patients treated with TJA were investigated. DVT patients (73 patients) were considered as the study group while non-DVT patients matched 1:4 according to anticoagulant type were considered as the control group (292 patients). These variables of potential risk factors for DVT included age, patient gender, individual weight status (BMI), poor lifestyle, comorbidities, preoperative hemoglobin (HB) level and analgesic use, site (knee or hip) and type of surgery, time of initiation of drug anticoagulation collection, ambulation time, blood transfusion and use of tranexamic acid (TXA), drainage, human serum albumin, and postoperative physical thromboprophylaxis. Postoperative physical thromboprophylaxis were evaluated by survival analysis with $95 \%$ confidence intervals expressed as p-values and dominance ratios. Results: Of the 5326 patients who underwent TJA, $73(0.48 \%)$ had DVT, $0.71 \%$ had total knee arthroplasty (TKA), and $0.24 \%$ had total hip arthroplasty. Risk factors associated with DVT included TKA (compared with THA), advanced age ( $>70$ years), drainage use, and delayed postoperative bed release (272 hours). The results showed that the incidence of DVT was relatively low in patients undergoing TJA $(0.48 \%)$. Risk factors associated with an increased risk of DVT included TKA (compared with THA), old age, drainage use, and delayed postoperative bed discharge. Individualized and more effective risk stratification protocols for post-TJA anticoagulation in the Chinese population may be needed in the future.

\subsection{Objectives}

The objectives of this review study are to:

i. Exploring care measures to prevent thrombosis after hip arthroplasty in the elderly

ii. Discussing the advantages and disadvantages of different nursing and prevention programs to prevent thrombosis after hip replacement in the elderly

iii. Discussing how to better construct a preventive program for postoperative care of the elderly hip arthroplasty prevent thrombosis

iv. Recommendations for the current care plan for elderly patients after hip arthroplasty to prevent thrombosis 


\section{Methods}

A comprehensive narrative review of the literature related to thromboprophylaxis care protocols for elderly patients after hip arthroplasty was conducted using academic databases such as Zhiwang, Wanfang, and Vipul. The researchers used various appropriate combinations of search terms, keywords, and phrases, such as "geriatric care, hip arthroplasty, thrombosis prevention, nursing care, DVT, orthopedic surgery, and elderly patients." Relevant articles that met the objectives of the review were retrieved and utilized in the study. In addition, additional articles obtained from the references of the selected articles that were relevant to the topic and objectives of the study were also reviewed.

\section{Findings}

\subsection{General Comprehensive Nursing Care}

\subsubsection{Bedside Nursing}

After the patient returned to the ward safely after surgery, the nurse performed regular turning for him/her. In the supine position, the affected limb should be abducted by $10-20^{\circ}$ and externally rotated by $5-10^{\circ}$, so that the patient's hip joint can be flexed forward by $15-20^{\circ}$ and the knee joint is flexed by $5-10^{\circ}$. When changing to the prone position, put small soft pillows under both knees and always keep the affected limb in the abducted neutral position. If possible, put on thong shoes to fix the patient's position. Be careful not to take the affected side to avoid secondary injury. For the affected limb, the use of hot water bags and ice packs for treatment is prohibited. Hot and cold therapy is not recommended for DVT patients to avoid aggravation of the disease and increase the patient's pain (Leng, 2019).

\subsubsection{Exercise Care}

Exercise rehabilitation is the least costly intervention to prevent deep vein thrombosis. As a nursing staff, they should actively use their knowledge of relevant postoperative orthopaedic care to develop individualized exercise programs for patients with their physicians and to properly demonstrates and inform elderly patients of postoperative hip exercises. Examples include common ankle pump exercises, straight leg lifts, joint flexion exercises, including ankle up and down and around exercises, and hip elevation exercises. If the patient is unable to perform them alone, the nurse will properly instruct the family to cooperate with them and adhere to the daily exercises. In addition, the patient should promptly give lower limb massage to promote blood circulation after surgery, three times a day for 15 min. active exercises included: 1). ankle pump: the blood circulation in the blood vessels may accelerate by muscle compression to prevent thrombosis. 2). Straight leg lift: mainly exercises the muscle strength of quadriceps. The normal leg is raised while the heel is $10 \mathrm{~cm}$ from the bed, and this position is maintained for 10s; the affected leg is tried to be raised for $10 \mathrm{~s}$. 5min each time, increasing to $15-20 \mathrm{~min}$ according to the patient's heart rate and general condition; the patient is told to do his best. 3). Joint flexion exercises: mainly to increase joint flexibility, hold next to the bed, the affected limb slowly flexed, gradually, about 20 times a day. The above exercises should stope when the patient felt fatigue or pain and an appropriate rest was not performed (Zhao, 2016).

\subsubsection{Drug Prophylaxis}

Regular heparin, Low molecular weight heparin (LMWH) and anticoagulant drugs can be used for the prevention of DVT after hip surgery. Although LMWH can prevent thrombosis in patients with DVT, it inevitably produces side effects such as thrombocytopenia and osteoporosis, and long-term injection can also lead to skin damage. Compared with LMWH, rivaroxaban oral drug shows its unique advantages. In a study by Liu Minghui (Liu, 2013), it was pointed out that compared with LMWH, rivaroxaban not only has similar anticoagulant effects, but also does not require continuous monitoring of clotting time, is easy to use, and has significant advantages in terms of medication adherence. It is recommended that rivaroxaban tablets will be given orally in a timely manner after orthopedic hip surgery in the elderly (Cao, 2010). In their study of clinical practice findings for the prevention of DVT noted that the data on rivaroxaban being more effective in the prevention of elderly orthopedic postoperative patients compared to other drugs were consistently supported in the prevention of DVT. Efficacy and outcome indicators such as thrombosis rate, bleeding rate, and patient satisfaction showed that the rivaroxaban group was significantly better than the LMWH group.

\subsection{Mechanical Physical Care}

\subsubsection{Plantar Venous Pump}

The venous pump is specially designed for DVT, which is based on the principle of Gardner's theory, by simulating the downward compression of the plantar venous plexus by increasing gravity during walking, intermittent compression is systematically implemented on the bottom of the foot, forcing the blood on the bottom of the foot to pass through the compression, increasing the blood flow rate of the lower limb and 
promoting blood circulation. Traditional massage methods are affected by external factors such as uneven force, thus making the massage ineffective, however, the venous pump is a medical device that can use high-tech highly intelligent sensing system model analysis to set parameters to adjust the time after analysis of patients with different conditions, and inflatable massage for patients distal end to proximal end in a gradual manner, this medical device is both convenient and safe. Pan Linxiang (Pan, 2018) et al.. gave the observation group of patients in their study a 30-minute plantar venous pump treatment every $8 \mathrm{~h}$. The results showed that there was a significant reduction in the incidence of veins for outcome indicators in this study, which should be vigorously promoted for clinical application.

\subsubsection{Pneumatic Pump}

Pneumatic pump is a kind of medical device different from plantar vein pump. The main method of use is strapped to the outside of the patient's limb, through the use of mechanical air pressure to squeeze the limb, so that the limb blood continuous accelerated circulation to get the massage effect. In addition, this device uses intermittent air pressure to promote fibrinolysis and reduce platelets aggregation, effectively avoiding blood clotting and achieving good results in thrombosis prevention (Wang, 2019). The results of studies by Chen Lu (Chen, 2021) and Dai Huiyan (Dai, 2019) both supported the effectiveness of pneumatic compression pumps in preventing DVT after orthopedic hip fractures in the elderly. In particular, the use of pneumatic devices intraoperatively can play a role in preventing thrombosis.

\subsubsection{Medical Compression Stockings}

Medical compression stockings, also known as varicose veins stockings, are simple and generous in design; they are quick and easy to use, similar to wearing stockings. Medical compression stockings are made of a special fabric, and when worn, they are pressurized in layers, decreasing pressure from the distal to the proximal end of the body in small to large spiral, giving pressure to the blood vessels, causing the blood at the end of the body to return quickly to the center, preventing thrombosis and improving the slow flow of blood in patients (Jin, 2017). Before applying medical compression stockings to the patient, the nursing staff measured the maximum leg circumference by means of a soft ruler, and recommended the appropriate compression stocking grade by the measured value to ensure moderation and tightness. The stockings were worn as early as possible after orthopedic surgery without interfering with the patient's activities, and could be worn for a long time even after discharge from the hospital. In addition, Meng Qingling et al.. (Meng, 2017) concluded in a study that although the effect of elastic bandage is the same as that of elastic stockings, elastic stockings are more lifelike, simple to get to started quickly and conveniently, less damaging to the skin, more comfortable to wear, and more effective.

\subsection{Psychological Care}

As hip fractures occur in the elderly mostly as a result of accidents, they increase the economic pressure on the family during hospitalization, take up the children's working time, and have a longer postoperative recovery cycle time compared to the average patient. If psychological problems occur, it will have a negative impact on the recovery and prognosis of patients (Sun, 2018). Therefore, it is necessary to pay attention to the psychological state of patients in postoperative care and intervene in a timely manner. In this regard, Lv Jianxiu et al., (Lv, 2021) conducted a study in which they selected 66 patients suffering from deep vein thrombosis and randomly divided them into 2 groups using a blinded method. The control group underwent conventional treatment and subsequently, on the basis of conventional treatment in the control group, the study group used different psychological care and the final results of the study showed a reduction in the incidence of venous thrombosis and a reduction in the incidence of other complications such as urinary retention and pulmonary swelling, and the patients rated all aspects of their care higher than the control group. This indicates that the application of psychological care interventions in the postoperative care of hip fracture patients is effective and reducing the incidence of adverse reactions, so nurses should actively respond to psychological problems in the postoperative care of patients, thus accelerating their recovery.

\subsection{Improving Nurses' Knowledge of Blood Clots}

DVT of the lower extremity is a common complication in elderly postoperative orthopedic hip fracture patients, and in such patients, single method preventive measures are often poor. It is essential for clinical nursing workers to improve nursing knowledge of DVT prevention after orthopedic hip surgery in the elderly. Lau et al. (Lau, 2017; Wang, 2020) explored the effectiveness of two different web-based bedside nurse education tools in venous thromboembolism prophylaxis practice and proposed a method to improve nurses' knowledge of venous thromboembolism care in a comprehensive manner with the help of a web-based approach, which in turn can provide an opportunity to improve nursing knowledge of venous thromboembolism in the present We propose a web-based approach to improve nurses' knowledge of venous thromboembolism to provide a solid foundation for "defect-free" prevention of venous thromboembolism. Why is it important for nurses to improve their 
knowledge about DVT prevention? In a foreign survey, the professionalism and initiative of nurses can play an important role in the prevention of DVT in patients (Ding, 2009).

\section{Conclusion}

The results of this study showed that DVT as a preventable disease and to avoid this complication in patients, health care workers should pay attention to the systematic training of relevant knowledge and choose scientific prevention methods when caring for patients in order to better prevent the occurrence of complications such as DVT and improve the quality of life and prognosis of patients. In the domestic and international literature in recent years, it is easy to find that the clinical treatment of DVT is constantly advancing, such as oral rivaroxaban and the latest thrombolytic protocols. Nursing should also keep pace with the times and publish the nursing prevention guidelines and standardized preventive care measures for DVT as soon as possible, which will facilitate the effective implementation of DVT prevention in hospitals and elderly patients in orthopedic departments.

\section{Recommendations}

The advantages of traditional general comprehensive care are: i) economical and convenient; ii) effective; and iii) simple and easy for patients to understand. The disadvantages are: i) the need for professional guidance from the nursing staff; ii) the inability to ensure patient compliance; and iii) the degree of cooperation from the family. The advantages of mechanical physical care are: 1). easy to use and convenient; 2). strong treatment effect compared with traditional treatment methods; 3 ). personalized treatment. The disadvantages are: 1). some devices are expensive; 2). increase the risk of skin damage to patients; 3 ). lack of integration of treatment effect detection equipment. The advantages of psychological care are: 1). enhance the confidence of patients to promote recovery. The disadvantages are: 1). comprehensive tertiary hospitals have heavy workloads and cannot always pay attention to patients' psychological conditions. Therefore, it is clinically necessary to combine the above multiple care modalities for postoperative care of patients to prevent postoperative complications such as DVT. Age, as one of the most important risk factors, deserves attention and thus individualized care.

\section{References}

Cao, Y. B., Zhang, J. D., Shen, H., \& et al.. (2010). Rivaroxaban versus enoxaparin for thromboprophylaxis after total hip or knee arthroplasty: a meta-analysis of randomized controlled trials. Eur. J. Clin. Pharmacol, 66(11), 1099-1008. https://doi.org/10.1007/s00228-010-0889-z

Chen, L. (2021). Effect of pneumatic pump combined with nursing intervention on the prevention of lower limb deep vein thrombosis in patients after hip fracture surgery. Medical Equipment, 34(6), 158-159.

Chen, W.-Y, Wei, M., Pan, L., \& Fu, Q.-M. (2020). A meta-analysis of risk factors for deep vein thrombosis in patients with hip and knee prosthetic joint replacement in China. Modern Medicine, 7, 879-884.

Dai. H.-Y., Gao, R.-R., Xu, P., \& et al.. (2019). Efficacy analysis of pneumatic pump combined with low-molecular heparin calcium in preventing deep vein thrombosis after hip fracture surgery. Journal of Practical Clinical Medicine, 23(9), 24-26.

Ding, Y.-M. (2009). Survey analysis of nurses' awareness of the current situation of deep vein thrombosis. Contemporary Nursing, 15(2), 99-100.

Golomb, B. A., Chan, V. T., Denenberg, J. O., \& et al.. (2014). Risk marker associations with venous thrombotic events: a cross-sectional analysis. BMJ Open, 4(3), e003208. https://doi.org/10.1136/bmjopen-2013-003208

Jin, Y.-J., \& Li, H.-L. (2017). Assessment and analysis of the risk of deep vein thrombosis in bedridden neurosurgical patients. Chinese Journal of Practical Nursing, 33(1), 28-31.

Keenan, C. R., \& White, R. H. (2007). The effects of race/ethnicity and sex on the risk of venous thromboembolism. Curr Opin Pulm Med, 13(5), 377-383. https://doi.org/10.1097/MCP.0b013e3281eb8ef0

Lau, B.-D., Shaffer, D.-L., Hobson, D.-B., Yenokyan, G., Wang, J., Sugar, E. A., ... Haut, E. R. (2017, August). Effectiveness of two distinct web-based education tools for bedside nurses on medication administration practice for venous thromboembolism prevention: a randomized clinical trial. PLoS One, 12(8), e0181664. https://doi.org/10.1371/journal.pone.0181664

Leng, N., \& Cao, L.-Y. (2019). Effect of comprehensive care for elderly patients with hip fractures after surgery on the prevention of deep vein thrombosis in their lower limbs. Contemporary Medicine Series, 17(21), 255-256.

Li. L., He, L.-Y., Tian, Y.-C., \& et al.. (2016). Effect of nursing intervention on deep vein thrombosis after hip replacement. Journal of Hebei Medical University, 37(1), 75-77. 
Liu, M. F., Wang, Y. Q., Tian, X. Z., \& et al.. (2013). Comparison of rivaroxaban and low molecular heparin for prevention of thrombosis after internal fixation of hip fracture. Tianjin Medicine, (8), 823-824.

Liu, X.-Q. (2020). Advances in the prevention and care of postoperative deep vein thrombosis in orthopedics. Electronic Journal of Clinical Medicine Literature, 7(48), 191-192.

Lv, J.-X. (2021). Study on the application of psychological nursing intervention in the postoperative care of hip fracture patients. Psychological Monthly, 16(9), 117-118.

Meng, Q.-L., Tan, S.-C.-T., \& Ma, L. (2017). Clinical effects of compression bandage applied to postoperative patients with hip fracture. Continuing Medical Education, 31(12), 125-127.

National Bureau of Statistics. (2021). The Seventh National Population Census Main Data. [EB/OL]. Retrieved May 11, 2021, from http://www.gov.cn/xinwen/2021-05/11/content_5605871.htm

Pan, L.-X., \& Wu, J. (2018). Observation of nursing intervention effect of plantar venous pump for prevention of deep vein thrombosis after hip fracture surgery in the elderly. Electronic Journal of Practical Clinical Nursing, 3(41), 95.

Parmelee, D., Michaelsen, L. K., Cook, S., \& et al.. (2012). Team-based learning: a practical guide: AMEE guide no. 65. Med Teach, 34(5), e275-287. https://doi.org/10.3109/0142159X.2012.651179

Prisco, D., Cenci, C., Silvestri, E., \& et al.. (2014). Pharmacological prevention of venous thromboembolism in orthopaedic surgery. Clin Cases Miner Bone Metab, 11(3), 192-195. https://doi.org/10.11138/ccmbm/2014.11.3.192

Sun, J.-L., \& Li, Q.-Z. (2018). Analysis of the value of extended care intervention in 90 hip fracture patients. Genomics and Applied Biology, 37(3), 989-995.

Wang, H.-M. (2019). The application experience of comprehensive nursing intervention in the prevention of deep vein thrombosis after lower limb fracture surgery. China Practical Medicine, 14(6), 165-166.

Wang, Y., Ma, Y.-F., Deng, H.-B., Yang, X., Wang, L., Wang, X.-J., ... Wu, X.-J. (2020). Clustering analysis of hot common words in domestic venous thromboembolism nursing research. Chinese Journal of Nursing, 7 , 1001-1005.

Wang, Y.-H, Gu, W.-D., Zhang, Y.-P., \& et al.. (2016). The role of nursing intervention management in preventing lower limb deep vein thrombosis after hip fracture surgery in the elderly. Nursing Practice and Research, 13(21), 109-111.

Xu, H., Zhang, S.-Y., Xie, J.-W., Lei, Y.-T., Cao, G.-R., Chen, G., \& Pei, F.-X. (2019). Anested case-control study onthe risk factor so deep vein thrombosis for Chinese after total join tarthroplasty. Journal of Orthopaedic Surgery and Research, 141. https://doi.org/10.1186/s13018-019-1231-9

Xu, S.-J. (2017). Advances in deep vein thrombosis risk assessment and preventive care. Journal of Nursing, 32(7), 110-112.

Xu, Y.-F., \& Zhou, Y.-F. (2016). Continuity of care for patients with total hip arthroplasty. Journal of Liberation Army Nursing, 33(17), 50-52.

Yu. J. (2019). Exploration of nursing measures to prevent lower limb thrombosis in elderly patients with hip fracture. Anhui Medicine, 23(8), 1596-1599.

Zhao, X. (2016). Early warning nursing intervention for deep vein thrombosis in postoperative patients with lung cancer. Journal of Nursing, 31(2), 44-45.

Zheng, S. H. B., \& Lu, X. J. (2017). High-risk factors of deep vein thrombosis and the progress of preventive care. Contemporary Nurse, (2), 15-18.

Zhuang, S.-J. (2007). Emphasis on the prevention and treatment of perioperative deep vein thrombosis in elderly patients. Geriatrics and Health Care, (2), 76-78, 84.

\section{Copyrights}

Copyright for this article is retained by the author(s), with first publication rights granted to the journal.

This is an open-access article distributed under the terms and conditions of the Creative Commons Attribution license (http://creativecommons.org/licenses/by/4.0/). 